\title{
Grafting of alkoxyamine end-capped (co)polymers onto multi-walled carbon nanotubes
}

\author{
Xudong Lou, Christophe Detrembleur, Valérie Sciannamea, Christophe Pagnoulle and Robert Jérôme \\ Center for Education and Research on Macromolecules (CERM), University of Liège, Sart-Tilman, B6, 4000 \\ Liège, Belgium
}

\begin{abstract}
Multi-walled carbon nanotubes (MWNTs) have been successfully modified by polystyrene, poly( $\varepsilon^{*}$ caprolactone), and their block copolymers by addition reaction of the alkoxyamine-terminated precursors. Polymer-modified MWNTs are easily dispersed in good solvents for the grafted polymer, such as toluene and THF. This observation has been confirmed by TEM analysis. The grafting ratio of polystyrene chains at the surface of MWNTs depends on the polymer molecular weight.
\end{abstract}

Keywords: Multi-walled carbon nanotubes; Surface modification; Poly(E-caprolactone)

\section{Introduction}

Since their discovery in 1991 [1], carbon nanotubes (CNTs) are receiving steadily increasing attention because of their unique structural and electrical characteristics [2]. Moreover, they are potential modifiers for polymer matrices to upgrade their inherent properties [3-6]. However, preparation of polymer-CNTs hybrid materials strongly depend on the extent of the CNTs dispersion and the strength of the interfacial adhesion. It is the reason why the surface properties of CNTs have to be modified by the grafting of organic molecules, including polymer chains [7-11]. 'Grafting from' and 'grafting to' methods were recently reported for the covalent bonding of polymer onto CNTs. The 'grafting from' technique relies on the immobilization of initiators onto the tubes followed by surface-initiated polymerization and formation of a possibly dense polymer brushes. For instance, carbanions have been generated at the CNTs' surface by reaction with an anionic compound (sec-butyl lithium), followed by the surface-initiated polymerization of styrene [12]. According to a similar strategy, ATRP initiators have been attached to CNTs and poly( $n$-butyl methacrylate), poly(methyl methacrylate), and polystyrene chains have been grown from the surface [13-16]. However, this is a time-consuming procedure and the metal residue has to be removed before use. As an example of 'grafting to' technique, Qin et al. [16] have reported on the cycloaddition reaction of polystyrene- $\mathrm{N}_{3}$ onto CNTs. A multi-step process is, however, required for the synthesis of the azide end-capped polystyrene, and a long reaction time is an additional drawback. It is known that 'living' polystyrene end-capped by a TEMPO group can react with $\mathrm{C}_{60}$ according to a radical mechanism, with formation of a PS- $\mathrm{C}_{60}$ adduct [17-18]. This grafting strategy should be also effective in case of MWNTs rather than $\mathrm{C}_{60}$.

In this paper, alkoxyamine-terminated polystyrene, poly( $\varepsilon$-caprolactone), and their block copolymers have been prepared with controlled molecular weight and narrow molecular weight distribution. These (co)polymers have been reacted with multi-walled carbon nanotubes (MWNTs). This process is shown in Scheme 1. 
<smiles>CC(ON1C(C)(C)CCCC1(C)C)c1ccccc1CCOC(=O)c1ccccc1</smiles>

PS.TEMPO<smiles>CC(C)C(NOC(COC(=O)c1ccccc1)c1ccccc1)C(C)(C)C</smiles>

PS-NO<smiles>CC1(C)CCCC(C)(C)N1OC(COC(=O)CCCCCO)c1ccccc1</smiles><smiles>CC1(C)CCCC(C)(C)N1O[C@H](COCC(=O)O)c1ccccc1</smiles>

PCL-TEMPO

(PCL-b.PS)-TEMPO
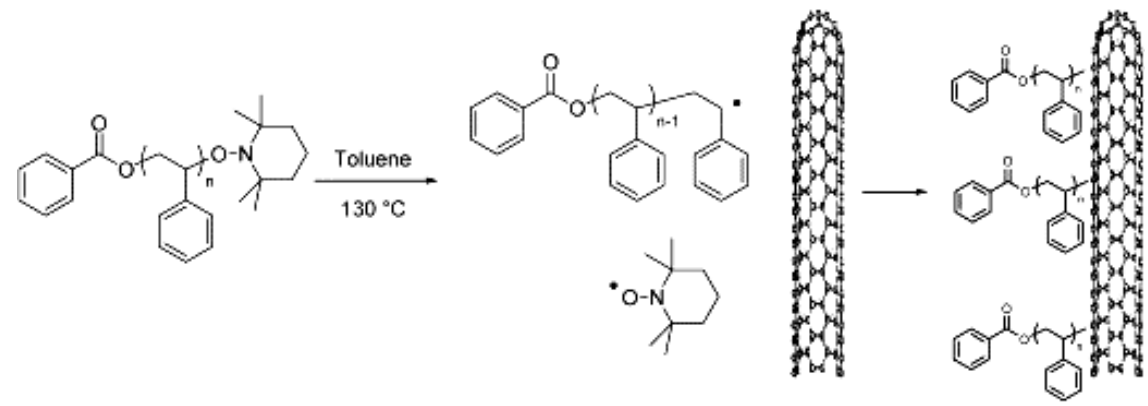

Scheme 1.

\section{Experimental part}

\subsection{Materials}

Commercially available CVD thick MWNTs (supplied by 'Nanocyl S.A.' Belgium; $50 \mu \mathrm{m}$ long with an average inner diameter of $6 \mathrm{~nm}$, an outer diameter of $25 \mathrm{~nm}$, and a purity higher than $95 \mathrm{wt} \%$ ) were used in this study without further purification. Styrene and $z$-caprolactone were dried over calcium hydride and distilled under reduced pressure just before use.

\subsection{Synthesis of alkoxyamine-terminated polymers}

PS-TEMPO [17] and PS-NO [19] (Scheme 1) were prepared by nitroxide mediated radical polymerization of styrene. TEMPO-terminated poly( $E$-caprolactone) (PCL-TEMPO) and (PCL- $b$-PS)-TEMPO were prepared (Scheme 2) as described elsewhere [21].<smiles>CC1CCCC(C)(C)N1OC(CO)c1ccccc1</smiles><smiles>O=C1CCCCCCO1</smiles><smiles>CCCON1C(C)(C)CCCC1(C)ON1c2ccccc2C1COC(=O)CCCCCO</smiles>

$130^{\circ} \mathrm{C} \mid$ Styrene<smiles>CC(C)C(C)(C)Cc1cccc2ccccc12</smiles>

(PCL-b-PS)-TEMPO

Scheme 2. 


\subsection{Functionalisation of MWNTs}

In a typical experiment, $20 \mathrm{mg}$ of MWNTs, $2.0 \mathrm{~g}$ of alkoxyamine end-capped polymer were added into a glass reactor and degassed by vacuum-nitrogen cycles (three times). Six millilitres of degassed toluene were added, and the mixture was heated at $130{ }^{\circ} \mathrm{C}$ under stirring for $24 \mathrm{~h}$. After cooling down to $25^{\circ} \mathrm{C}, 10 \mathrm{ml}$ of toluene were added, and the MWNTs were recovered by filtration with a $200 \mathrm{~nm}$ Teflon membrane and washed with toluene and THF. They were redispersed in THF and filtered again, in order to remove the non-grafted chains completely. This procedure was repeated at least four times until no polymer was extracted anymore.

\subsection{Characterization}

Thermogravimetric analysis (TGA) was carried out with a TA Instruments TGA Q500 thermal analyzer in the $25-500{ }^{\circ} \mathrm{C}$ range, at a $10^{\circ} \mathrm{C} / \mathrm{min}$ rate, under nitrogen TEM was performed with a Philips CM 100 apparatus, equipped with a Gatan $675 \mathrm{CCD}$ camera for digital imaging, at an accelerating voltage of $100 \mathrm{kV}$. A drop of MWNTs dispersion was deposited on a TEM copper grid (3.05 mm, 200 mesh) coated with a Formvar film, and the solvent was allowed to evaporate. Scanning electron microscopy (SEM) was performed with a Jeol JSM-840 A microscope. The sample was coated with platinum (sputtering with Balzers SCP-20 for $120 \mathrm{~s}$ under Argon).

\section{Results and discussion}

A series of alkoxyamine-terminated polymers shown in Scheme 1 have been prepared with the purpose to modify MWNTs. Samples of TEMPO end-capped polystyrene (PS-TEMPO) are listed in Table 1. They were prepared by controlled radical polymerization of styrene in the presence of benzoyl peroxide and TEMPO. Molecular weight is in the 3000-28,500 range, and polydispersity is approximately 1.25. PS-NO was prepared by controlled radical polymerization of styrene in the presence of $N$-tert-butyl- $\alpha$-isopropylnitrone and benzoyl peroxide as published elsewhere [19]. TEMPO-terminated poly( $\varepsilon$-caprolactone) (PCL-TEMPO) was prepared by living ring-opening polymerization of $k$-caprolactone initiated by aluminium isopropoxide in the presence of a hydroxyl containing alkoxyamine, 1 (Scheme 2) [20]. Furthermore, PCL-TEMPO is a macroinitiator for the controlled radical polymerization of styrene at $130{ }^{\circ} \mathrm{C}$, thus a precursor of PCL- $b$-PS block copolymers with a narrow molecular weight distribution [20].

Table 1. Molecular characteristics of TEMPO end-capped (co)polymers and reaction products with MWNTs at $130{ }^{\circ} \mathrm{C}$

\begin{tabular}{|l|l|l|l|l|l|}
\hline Entry & Polymer $^{\mathbf{a}}$ & $\boldsymbol{M}_{\mathbf{n}}^{\mathbf{b}} \times \mathbf{1 0}^{-\mathbf{3}}$ & $\boldsymbol{M}_{\mathbf{w}} / \boldsymbol{M}_{\mathbf{n}}$ & Time (h) & $\mathbf{G R}^{\mathbf{c}} \mathbf{( \% )}$ \\
\hline $1 \mathrm{a}$ & PS-TEMPO & 3.0 & 1.20 & 24 & 12 \\
\hline $1 \mathrm{~b}$ & PS-TEMPO & 3.0 & 1.20 & 43 & 12.5 \\
\hline $1 \mathrm{c}$ & PS-TEMPO & 17.5 & 1.25 & 24 & 20 \\
\hline $1 \mathrm{~d}$ & PS-TEMPO & 19.5 & 1.20 & 24 & 17 \\
\hline $1 \mathrm{e}$ & PS-TEMPO & 28.5 & 1.25 & 24 & 24 \\
\hline $1 \mathrm{f}$ & PS-NO & 12.0 & 1.40 & 24 & 14 \\
\hline $1 \mathrm{~h}$ & PCL-TEMPO & 7.0 & 1.20 & 24 & 25 \\
\hline $1 \mathrm{i}$ & PCL-TEMPO & 14.5 & 1.15 & 24 & 28 \\
\hline $1 \mathrm{j}$ & (PCL- $b$-PS)-TEMPO & 15.5 & 1.20 & 24 & 30 \\
\hline
\end{tabular}

Reaction conditions: $2.0 \mathrm{~g}$ of (co)polymer, $20 \mathrm{mg}$ of MWNTs in $6 \mathrm{ml}$ of toluene.

a TEMPO-terminated polymer.

${ }^{\mathrm{b}} M_{\mathrm{n}}$, GPC based on standard PS.

${ }^{\mathrm{c}} \mathrm{GR}$, grafting ratio of polymer based on TGA, defined as the weight ratio of the grafted polymer to the nanotubes. 
Table 1 reports on the molecular characteristics of the nitroxide end-capped polymers and the products of reaction with MWNTs. The reaction is conducted under stirring, in toluene at $130{ }^{\circ} \mathrm{C}$. Typically, 100 -fold wt excess of polymer was used with respect to carbon nanotubes. After reaction, the modified MWNTs were recovered by filtration through a $0.2 \mu \mathrm{m}$ Teflon membrane.

The polymer-grafted carbon nanotubes are easily dispersed in toluene and THF in contrast to neat MWNTs. Fig. 1(I) compares the stability of dispersions of PS- and PCL-grafted MWNTs in toluene, 7 days after ultrasonication. The visual aspect of the dispersions is drastically different, depending on whether the MWNTs have been surface modified or not. The reference dispersion (neat MWNTs) is unstable, a solid precipitate coexisting with a nearly transparent liquid supernatant. In sharp contrast, polymer modified MWNTs form stable dispersions, which makes the solvent deeply black. Less than 5\% of precipitation is observed. As an additional and more direct evidence for the PS grafting onto carbon nanotubes, the surface modified tubes were analyzed by TEM. A few drops of the MWNTs dispersion were deposited onto a carbon-coated copper grid, and the solvent was let to evaporate. Fig. 1(II) shows individual nanotubes rather than the densely entangled pristine MWNTs (Fig. 1(III)), all the other conditions being the same. PCL-grafted MWNTs were added to an $\mathrm{HCl}$ solution in 1,4-dioxane and stirred at $80{ }^{\circ} \mathrm{C}$ for $5 \mathrm{~h}$. The PCL was accordingly degraded by hydrolysis, and the MWNTs were collected by filtration and poured into toluene (Fig. 1(I)). No stability is observed anymore, which indicates that the PCL chains immobilized at the surface of the MWNTs form an effective steric barrier against agglomeration. 

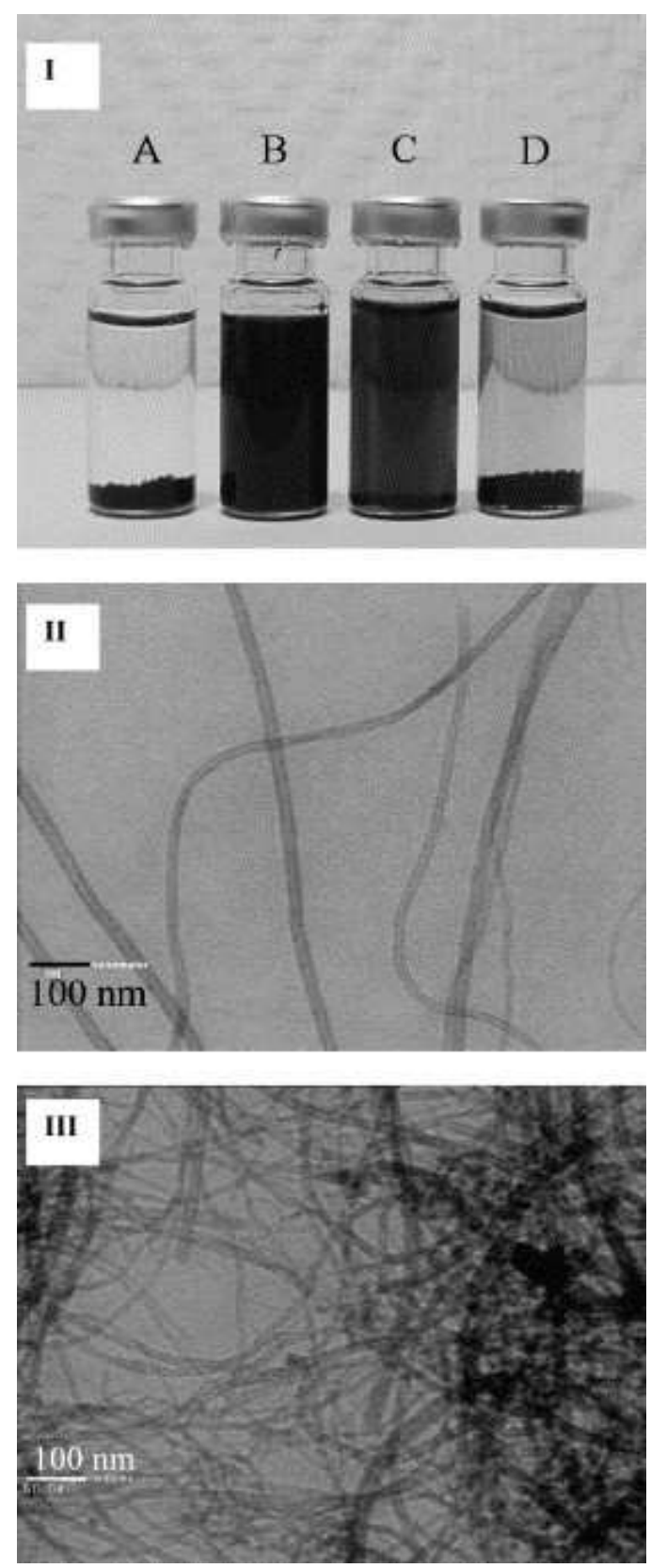

Fig. 1. (I) Observation of MWNTs dispersions in toluene, 7 days after ultrasonication (A) pristine MWNTs; (B) PS-grafted MWNTs; (C) PCL-grafted MWNTs; (D) PCL-grafted MWNTs after acid treatment. Transmission electron microscopy of (II) PS-grafted MWNTs and (III) pristine MWNTs.

The UV/vis absorption spectrum of PS-grafted MWNTs sample in THF at room temperature is shown in Fig. 2. The absorbance decreases gradually from UV to near-IR, similarly to the absorption spectrum of CNTs surface modified by different organic groups [21]. Because no stable dispersion of pristine MWNTs could be prepared (fast sedimentation of the tubes), no representative UV/vis spectrum could be recorded. Dispersions of PSgrafted MWNTs in THF are stable for months. Fig. 2 shows that the absorption at $400 \mathrm{~nm}$ remains essentially unchanged after 40 days. 

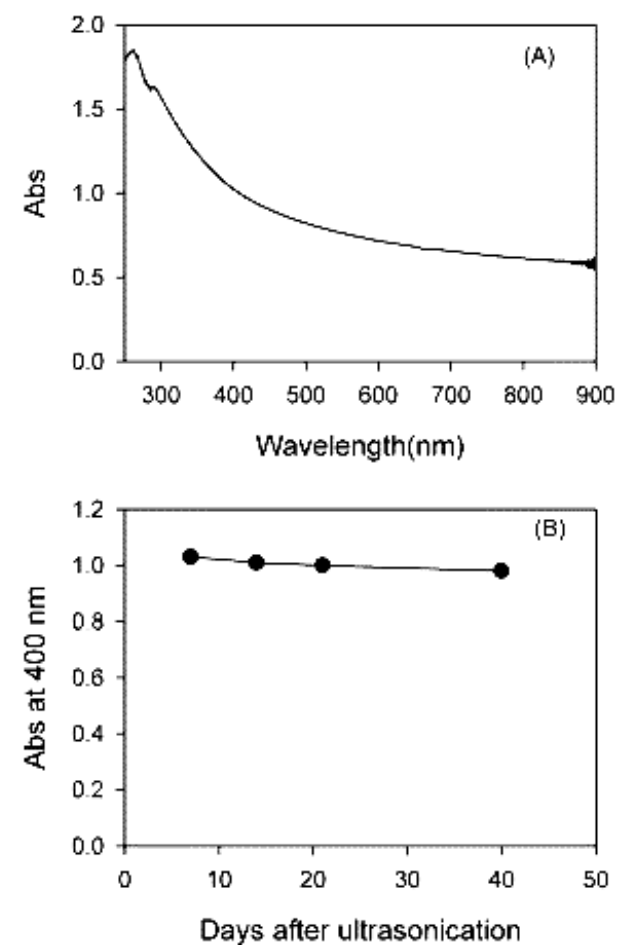

Fig. 2. (A) UV/vis absorption spectrum for PS-grafted MWNTs (1c in Table 1) in THF at room temperature; (B) Absorption at $400 \mathrm{~nm}$ versus time after ultrasonication.

Another alkoxyamine-terminated polystyrene, PS-NO in Scheme 1, have also been reacted with MWNTs under the same reaction conditions, leading to MWNTs dispersions as stable as the ones reported in case of PSTEMPO. The efficiency of the 'grafting to' method would thus be largely independent on the alkoxyamine endgroup.

The mechanism of the reaction is thought to be a radical addition, as described in case of $\mathrm{C}_{60}$ [17] and [18]. As shown in Scheme 1, polymeric radicals, that result from the cleavage of the $\mathrm{C}$-nitroxide bonds, can add to the double bonds of MWNTs.

The polymer chains grafted to MWNTs were selectively eliminated by heating (TGA) at $10{ }^{\circ} \mathrm{C} / \mathrm{min}$ under nitrogen. Fig. 3 (top) compares the TGA curves for pristine MWNTs, PS, and PS-grafted MWNTs. The pristine MWNTs are stable until $500^{\circ} \mathrm{C}$ with negligible weight loss. PS-grafted onto MWNTs is degraded at $300-$ $450{ }^{\circ} \mathrm{C}$, which is the same temperature as pure PS, leaving behind the thermally stable MWNTs. SEM of the degrafted MWNTs (Fig. 4) is reminiscent of the unmodified sample, which suggests that the thermal removal of the polymer does not change the carbon nanotubes structure. Fig. 3 (bottom) shows the thermal degradation of PCL and the two-step decomposition of PCL- $b$-PS previously attached to MWNTs, which confirms the presence of polymer chains onto the carbon nanotubes. 

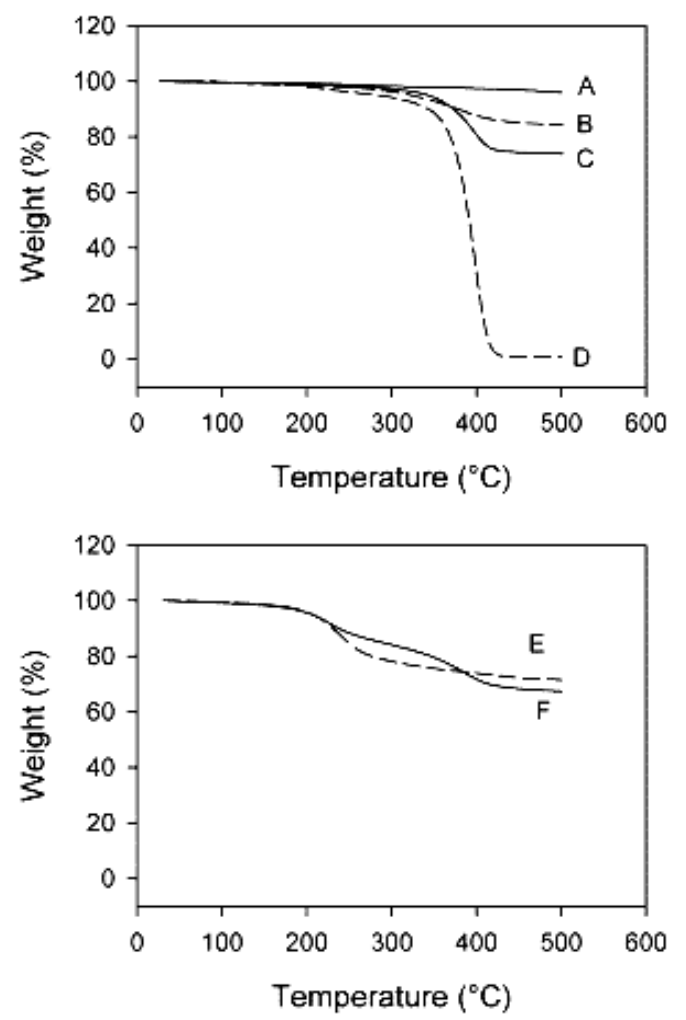

Fig. 3. Thermogravimetric analysis of polymers and MWNTs under nitrogen at $10^{\circ} \mathrm{C} / \mathrm{min}$. (A) Pristine MWNTs; (B) PS-grafted MWNTs (1a, in Table 1); (C) PS-grafted MWNTs (1e, in Table 1); (D) PS; (E) PCL-grafted MWNTs (1h, in Table 1); (F) PCL-b-PS-grafted MWNTs (1j, in Table 1).

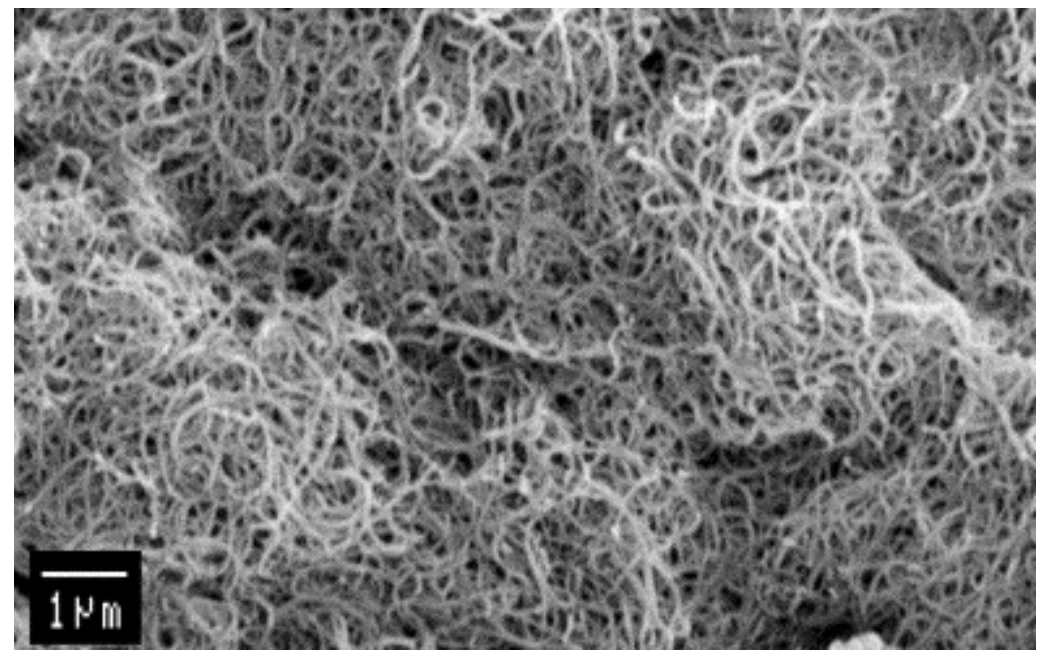

Fig. 4. SEM image of PS-grafted MWNTs after thermal degrafting (TGA scan up to $500^{\circ} \mathrm{C}$, under nitrogen).

The grafting ratio (GR), defined as the weight ratio of the grafted polymer to the nanotubes, was determined by TGA under nitrogen. Data are listed in Table 1. The reaction is complete within $24 \mathrm{~h}$ (or less) as confirmed by the comparison of entries $1 \mathrm{a}$ and $1 \mathrm{~b}$. As a rule, the grafting ratio increases with the PS molecular weight, which allows the number of chains per mg of MWNTs to be estimated. Fig. 5 shows the dependence of this number on the molecular weight of PS-TEMPO. It appears that the coverage of the MWNTs surface is close to completion. Longer polymer chains cover a larger surface, and the grafting density decreases. Control of the PS-TEMPO molecular weight is actually a way to control the grafting density, which is promising for tuning the properties of modified MWNTs, thus the properties of MWNTs based nanocomposites. 


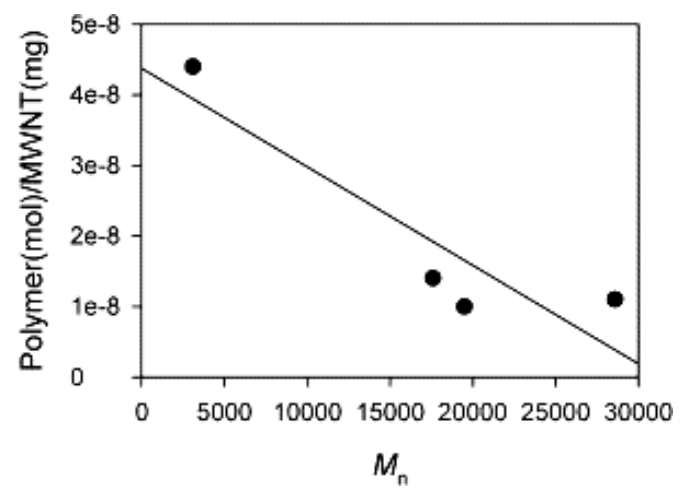

Fig. 5. Dependence of PS grafting density on $M_{n}$ of PS-TEMPO.

In conclusion, MWNTs have been successfully modified by PS, PCL, and PCL- $b$-PS copolymer as result of the addition reaction of the parent polymeric radicals. Grafted MWNTs are easily dispersed in good solvents for the grafted polymers, e.g. toluene and THF. This observation has been confirmed by TEM. The control of molecular weight of the grafted chains is an easy way to control the grafting density of the carbon nanotubes. Because a variety of polymers (polystyrenes, polydienes, polyacrylates, etc.) have been prepared by nitroxide mediated living radical polymerizations [22], all these polymers with controlled molecular characteristics can be grafted onto carbon nanotubes.

\section{Acknowledgements}

The authors are indebted to the 'Région Wallonne' for support in the frame of the 'Nanotechnologies' programme ENABLE. CERM also thanks the 'Belgian Science Policy' for financial support in the frame of the 'Interuniversity Attraction Poles Programme (PAI V/03) - Supramolecular chemistry and supramolecular catalysis' C.D. is “Chercheur Qualité” by the FNRS, Belgium.

\section{References}

[1] Iijima S. Nature 1991;354:56

[2] Ajayan PM. Chem Rev 1999;99:1787.

[3] Qian D, Dickey EC, Andrew R, Rantell T. Appl Phys Lett 2000; 76:2868.

[4] Kumar S, Dang TD, Arnold FE, Bhattacharyya AR, Min BG, Zhang X, Vaia RA, Park C, Adams WW, Hauge RH, Smalley RE, Ramesh S, Willis PA. Macromolecules 2002;35:9039.

[5] Geng H, Rosen R, Zheng B, Shimoda H, Fleming L, Liu J, Zhou O. Adv Mater 2002; 14:1387.

[6] Zengin H, Zhou W, Jin J, Czerw R, Smith DW, Echegoyen L, Carroll DL, Foulger SH, Ballato J. Adv Mater 2002;14:1480.

[7] Hirsch A. Angew Chem Int Ed 2002;41:1853.

[8] Sun YP, Fu K, Lin Y, Huang W. Acc Chem Res 2002;35:1096.

[9] Liu J, Rinzler AG, Dai H, Hafner JH, Bradley RK, Boul PJ, Lu A, Iverson T, Shelimov K, Huffman CB, Rodriguez-Macias F, Shon Y, Lee TR, Colbert DT, Smalley RE. Science 1998;280:1253.

[10] Sano M, Kamino A, Okamura J, Shinkai S. Langmuir 2001;17:5125.

[11] Shaffer MSP, Koziol K. Chem Commun 2002;18:2074.

[12] Viswanathan G, Chakrapani N, Yang H, Wei B, Chung H, Cho K, Ryu CY, Ajayan PM. J Am Chem Soc 2003; $125: 9258$.

[13] Qiu S, Qin D, Ford WT, Resasco DE, Herrera JE. J Am Chem Soc 2004;126:170.

[14] Yao Z, Braidy N, Botton GA, Adronov A. J Am Chem Soc 2003;125:16015.

[15] Kong H, Gao C, Yan D. J Am Chem Soc 2004;126:412.

[16] Qin S, Qin D, Ford WT, Resasco RE, Herrera JE. Macromolecules 2004;37:752.

[17] Okamura H, Terauchi T, Minoda M, Fukuda T, Komatsu K. Macromoleucles 1997;30:5279.

[18] Ford WT, Lary AL, Mourey TH. Macromolecules 2001;34:5819.

[19] Detrembleur C, Sciannamea V, Koulic C, Claes M, Hoebeke M, Jérôme R. Macromolecules 2002;35:7214.

[20] Hawker CJ, Hedrick JL, Malmstrom EE, Trollsas M, Mecerreyes D, Moineau G, Dubois Ph, Jérôme R. Macromolecules 1998;31:213.

[21] Hill DE, Lin Y, Rao AM, Allard LF, Sun YP. Macromolecules 2002; 35:9466.

[22] Hawker CJ, Bosman AW, Harth E. Chem Rev 2001; 101:3661. 